\title{
Risk factors of brain metastasis of lung squamous cell carcinoma: a retrospective analysis of 188 patients from single center
}

\author{
Bo Li, Yanwei Liu, Shuai Liu, Xuenan Gu and Xiaoguang Qiu*
}

\begin{abstract}
Background: To explore risk factors and the efficacy of treatment strategies for brain metastasis (BM) in squamous cell carcinoma (SCC) of the lung.

Methods: The clinical data of 188 pathologically confirmed as squamous cell carcinoma or adenosquamous carcinoma patients were studied retrospectively. Factors including age (<60 vs. $\geq 60$ ), gender, stage at diagnosis, T status ( $(11-2$ vs. T3-4), N status (N0-1 vs. N2-3), histology (squamous vs. adenosquamous), smoking history (non-smoker vs. current smoker) and serum tumor markers (normal vs. elevated) were analyzed.

Results: The incidence of BM was 19.1\% (36/188) in our cohort. Patients who were female $(p=0.005)$, had advanced disease at diagnosis $(p<0.001)$, had adenosquamous carcinoma histology $(p=0.033)$ or had elevated serum level of CEA at diagnosis $(p<0.001)$ had significantly higher incidence of BM. In multivariate analysis, female $(p=0.034$, $H R=18.874)$ and elevated serum level of CEA at diagnosis $(p=0.009, H R=19.824)$ were independent risk factors of BM. BM patients who received additional systemic therapy after local therapy had significantly longer post-BM survival than those who received local therapy only $(p=0.004, \mathrm{HR}=0.058)$. Gemcitabine/platinum-containing regimen (GP) and taxans/platinum-containing regimen (TP) led to comparable brain-metastasis-free survival (BMFS) $(p=0.10)$.

Conclusions: Females and patients with elevated serum level of CEA at diagnosis had a higher risk of developing BM. The following systemic therapy after local therapy prolonged the survival of BM patient, but the efficacy of GP and TP was comparable in terms of preventing BM.
\end{abstract}

Keywords: Brain metastasis, Chemotherapy, Non-small cell lung cancer, Risk factor, Squamous cell carcinoma

\section{Background}

The brain is one of the most common distant metastasis sites in non-small cell lung cancer (NSCLC). Up to 40\% of NSCLC patients develop brain metastasis (BM) during the course of disease [1-4]. BM is associated with a poor quality of life (QOL) and a dismal prognosis; without treatment, the median overall survival (OS) for BM patients is only 4-7 weeks [5-7]. In patients with small cell lung cancer (SCLC), prophylactic cranial irradiation (PCI) is effective in decreasing the incidence of $\mathrm{BM}$ and improving OS $[8,9]$. PCI has therefore been studied in

\footnotetext{
*Correspondence: ttyy6611@126.com

Department of Radiation Oncology, Beijing Tiantan Hospital affiliated to Capital Medical University, No. 6, Tiantan Xili, Dongcheng District, Beijing 100050, China
}

NSCLC populations. While it decreases the incidence of histological subtypes in these studies. Furthermore, characteristics other than the incidence of $\mathrm{BM}$ in SCC were not examined. There is therefore a need for comprehensive studies specifically addressing the clinical features of BM in SCC.

Local therapies such as whole brain radiation therapy (WBRT), stereotactic radiosurgery (SRS), and surgical 
resection are the standard of care for patients with BM. However, most patients die from systemic disease rather than intracranial failure. Systemic treatment with novel agents, such as tyrosine kinase inhibitors (TKIs) and pemetrexed, has been shown to be beneficial in patients with BM [14-18]. They could not only shrink the intracranial lesions, but also prolong the survival. But the efficacy was usually seen in patients with non-SCC histology. By far, none agent has previously been studied in SCC with BM. To better characterize the clinical features of BM in SCC and to investigate effective treatment strategy, we conducted a retrospective study that included 188 patients.

\section{Methods}

\section{Patients}

The clinical data of 188 consecutive patients diagnosed between September 1999 and December 2013 with pathologically confirmed SCC of the lung, were selected from the database and analyzed retrospectively. Before treatment, baseline assessments including blood routine test, biochemical examination, and enhanced-contrast computed tomography $(\mathrm{CT})$ and/or magnetic resonance imaging (MRI) were conducted. The treatment strategy was determined based on the stage at diagnosis. Principally, systemic therapy of platinum-based doublet was recommended post-operatively for patients with operable stage IB to IIIA disease. In fact, it was becoming routine recommendation since 2006, when concrete evidence has been obtained from studies concerning adjuvant chemotherapy. For patients with stage IIIB to IV disease, chemotherapy was recommended. All patients received routine follow-up every 3 to 6 months after the completion of therapy.

Enhanced-contrast MRI was used for BM screening if there was no contraindications. Otherwise, enhancedcontrast CT was considered. At the time of diagnosis, MRI was conducted routinely for all patients. During the time of follow-up, MRI was conducted every 3-6 months. If symptoms of CNS metastasis presented during the follow-up interval, MRI was conducted immediately. Radiation techniques such as SRS was reserved for patients with three or less BM lesions. Otherwise, WBRT would be considered. After the completion of radiotherapy, systemic therapy was recommended routinely. The study protocol was reviewed and approved by the institutional review boards and ethics committees of Beijing Tiantan Hospital affiliated to Capital Medical University. All patients provided informed consent prior to their inclusion in the study according to the Declaration of Helsinki.

\section{EGFR mutation testing}

Amplification refractory mutation system (ARMS) was employed to analyze EGFR mutations in paraffin-embedded tissue sections. Briefly, DNA of original tumor tissue, which was obtained from the slides under a dissecting microscope, was isolated with a QIAamp DNA Mini Kit (Qiagen Inc., Valencia, CA, USA). Then, ADx EGFR Mutations Detection Kit (Amoy Diagnostics, Xiamen, China) was employed for EGFR mutations detection. ABI 7500 (Applied Biosystems, Foster City, CA, USA) real-time polymerase chain reaction system was recruited for the assay according to the manufacturer's protocol.

\section{Statistical analysis}

IBM SPSS Statistics 19.0 software was used for data analysis. The Kaplan-Meier method was used to estimate survival and the log-rank test was used to detect difference of survival curves. OS was defined as the time from diagnosis to the patients' death of any reason or the last follow-up visit. Brain-metastasis-free survival (BMFS) was defined as the time from diagnosis to the documented BM. Cox regression was employed for multivariate analysis. The incidence of BM between patients with different risk factors was compared using chi-squared test.

\section{Results \\ Patient characteristics}

Patient characteristics were detailed in Table 1 . Of the 188 patients enrolled in the study, $86.7 \%$ were male and $13.3 \%$ were female. The median age at diagnosis was 63 years (range, $32-89$ years). At the time of diagnosis, $7.9 \%$ of patients had stage I, $12.8 \%$ had stage II, $51.1 \%$ had stage III and $28.2 \%$ had stage IV disease. In terms of histology, 93.1\% had SCC and 6.9\% had adenosquamous carcinoma. In terms of smoking status, $23.9 \%$ were nonsmokers, $71.3 \%$ were current smokers and the smoking history was unknown for 4.8\%. EGFR mutation status was analyzed in 33 patients. Twenty-eight patients had wild-type EGFR, 3 had a mutation at exon 19 and 2 had a mutation at exon 21 .

\section{Clinical factors related to BM}

Thirty-six patients had documented BM, of which 16 were synchronous and 20 were metachronous (Table 2). Among patients with BM, 24 had one intracranial lesion, 5 had 2-3 lesions, and 7 had $>3$ lesions. To explore the factors related to the incidence of BM, we analyzed age ( $<60$ vs. $\geq 60$ ), gender (male vs. female), stage at diagnosis, T status (T1-2 vs. T3-4), $\mathrm{N}$ status ( $\mathrm{N} 0-1$ vs. $\mathrm{N} 2-3$ ), histology (squamous vs. adenosquamous), smoking history (non-smoker vs. current smoker) and serum levels of tumor markers including carcinoembryonic antigen (CEA), neuron-specific enolase (NSE), cytokeratin 19 fragments (Cyfra21-1), and squamous cell carcinoma antigen (SCC). As shown in Table 1, patients who were female $(p=0.002)$, had advanced disease at diagnosis 
Table 1 Patient characteristics

\begin{tabular}{|c|c|c|c|c|c|}
\hline Variables & No. & $\%$ & No. of BM & Incidence of BM & $P$ value \\
\hline Median age (years) & $63(32-89)$ & & 36 & 19.1 & \\
\hline Age & & & & & 0.077 \\
\hline$<60$ & 79 & 42.0 & 20 & 25.3 & \\
\hline$\geq 60$ & 109 & 58.0 & 16 & 14.7 & \\
\hline Gender & & & & & 0.002 \\
\hline Male & 163 & 86.7 & 27 & 16.6 & \\
\hline Female & 25 & 13.3 & 9 & 36.0 & \\
\hline Stage & & & & & $<0.001$ \\
\hline I & 15 & 7.9 & 1 & 6.7 & \\
\hline$\|$ & 24 & 12.8 & 1 & 4.2 & \\
\hline III & 96 & 51.1 & 12 & 12.5 & \\
\hline IV & 53 & 28.2 & 22 & 41.5 & \\
\hline T status & & & & & 0.36 \\
\hline $\mathrm{T} 1-2$ & 121 & 64.4 & 25 & 20.6 & \\
\hline T3-4 & 67 & 35.6 & 11 & 16.4 & \\
\hline N status & & & & & 0.85 \\
\hline No-1 & 65 & 34.5 & 13 & 20.0 & \\
\hline $\mathrm{N} 2-3$ & 123 & 65.5 & 23 & 18.6 & \\
\hline EGFR status & & & & & - \\
\hline Wild-type & 28 & 84.8 & 5 & 17.9 & \\
\hline Exon19 mutation & 3 & 9.1 & 0 & 0 & \\
\hline Exon 21 mutation & 2 & 6.1 & 0 & 0 & \\
\hline Histology & & & & & 0.033 \\
\hline Squamous cell carcinoma & 175 & 93.1 & 32 & 18.3 & \\
\hline Adenosquamous carcinoma & 13 & 6.9 & 4 & 30.8 & \\
\hline Smoking history & & & & & $0.088^{a}$ \\
\hline Non-smoker & 45 & 23.9 & 12 & 26.7 & \\
\hline Current-smoker & 134 & 71.3 & 23 & 17.2 & \\
\hline Unknown & 9 & 4.8 & 1 & 11.1 & \\
\hline CEA & & & & & $<0.001$ \\
\hline Normal & 126 & 67.1 & 14 & 11.1 & \\
\hline Elevated & 62 & 32.9 & 22 & 35.5 & \\
\hline NSE & & 79 & & & 1.0 \\
\hline Normal & 89 & 47.3 & 17 & 19.1 & \\
\hline Elevated & 99 & 52.7 & 19 & 19.2 & \\
\hline Cyfra21-1 & & & & & 0.13 \\
\hline Normal & 43 & 22.8 & 11 & 25.5 & \\
\hline Elevated & 145 & 77.2 & 25 & 17.2 & \\
\hline SCC & & 80 & & & 0.68 \\
\hline Normal & 95 & 50.5 & 14 & 14.7 & \\
\hline Elevated & 93 & 49.5 & 12 & 12.9 & \\
\hline
\end{tabular}

Abbreviations: EGFR Epidermal growth factor receptor, BM Brain metastasis, CEA Carcinoembryonic antigen, NSE Neuron-specific enolase, Cyfra21-1 Cytokeratin 19 fragments, SCC Squamous cell carcinoma antigen

${ }^{a}$ patients with unknown smoking history were excluded 
Table 2 Characteristics of patients with BM

\begin{tabular}{|c|c|c|}
\hline Variables & No. & $\%$ \\
\hline \multicolumn{3}{|l|}{ BM } \\
\hline Synchronous & 16 & 44.4 \\
\hline Metachronous & 20 & 55.6 \\
\hline \multicolumn{3}{|l|}{ RPA } \\
\hline । & 8 & 22.2 \\
\hline$\|$ & 20 & 55.6 \\
\hline III & 8 & 22.2 \\
\hline \multicolumn{3}{|l|}{ GPA } \\
\hline $0-1$ & 10 & 27.8 \\
\hline $1.5-2.5$ & 17 & 47.2 \\
\hline 3 & 4 & 11.1 \\
\hline $3.5-4$ & 5 & 13.9 \\
\hline \multicolumn{3}{|l|}{ Number of BM } \\
\hline 1 & 24 & 66.7 \\
\hline $2-3$ & 5 & 13.9 \\
\hline$>3$ & 7 & 19.4 \\
\hline \multicolumn{3}{|c|}{$\begin{array}{l}\text { Presence of systemic metastasis } \\
\text { at the time of BM }\end{array}$} \\
\hline No & 9 & 25.0 \\
\hline Yes & 27 & 75.0 \\
\hline \multicolumn{3}{|l|}{ Treatments after BM } \\
\hline Local therapy only & 15 & 41.6 \\
\hline Local + systemic therapy & 21 & 58.4 \\
\hline \multicolumn{3}{|l|}{ Local therapy } \\
\hline SRS & $27(15)^{a}$ & 75.0 \\
\hline WBRT & $9(6)$ & 25.0 \\
\hline
\end{tabular}

Abbreviations: BM Brain metastasis, RPA Recursive partitioning analysis, GPA Graded prognostic assessment, WBRT Whole brain radiation therapy, SRS Stereotactic radiosurgery

${ }^{a}$ Number in the blanket was number of patients receiving additional systemic therapy after local therapy

$(p<0.001)$, had adenosquamous carcinoma histology $(p=0.033)$, or elevated serum level of CEA $(p<0.001)$ had a significantly higher incidence of BM. Whilst other factors were not significantly related to the incidence of BM. As BM was not documented in patients with EGFR mutations, the difference in incidence according to EGFR mutation status was not analyzed.

In addition to the incidence of BM, BMFS was employed to evaluate the risk of BM for given patients. For the entire group, the median BMFS was not reached. Single variant analysis demonstrated that female $(p=0.010)$, patients with advanced disease $(p=0.019)$ or elevated serum level of CEA $(p<0.001)$ had significantly shorter median BMFS than their counterparts. However, only females $(p=0.034$, hazard ratio $[\mathrm{HR}]=18.874)$ and elevated serum level of CEA at diagnosis $(p=0.009, \mathrm{HR}=19.824)$ were proved to be independent risk factors of BM in multivariate analysis.

\section{Survival analysis}

In terms of post-BM treatments, 27 received SRS and 9 received WBRT. Sequentially, additional systemic therapy was administrated in 21 patients. The median postBM survival for patients with BM was 18 months (95\% CI 12-23 months). Recursive partitioning analysis (RPA) and graded prognostic assessment (GPA) score are both valuable parameters that were used for prognosis evaluation of BM patients. As it was shown, the median postBM survival for RPA classes I-III were: 24 months, not reached and 3 months, respectively (Fig. 1). The statistical difference was significant whenever it was calculated in single variant $(p<0.001)$ or multivariate analysis $(p=0.013$, hazard ratio HR $=23.379)$. However, the difference of median post-BM survival according to the graded prognostic assessment (GPA) score did not reach significance. Survival analysis also found that, patients who received systemic therapy after the completion of local therapy had a longer post-BM survival than those who received local therapy only ( 24 months vs. 18 months, $p=0.084$ ) (Fig. 2). But the difference was marginal. In multivariate analysis, additional systemic therapy after local therapy was an independent factor that indicated better post-BM survival $(p=0.004$, hazard ratio $\mathrm{HR}=0.058$ ) (Table 3 ).

Besides, additional analysis was conducted to explore the role of different systemic regimens in BM patients. In patients who received systemic therapy after BM, survival analysis found there was a tendency that patients who received gemcitabine/platinum-containing (GP) regimen $(n=11)$ had longer post-BM survival than those who received taxans/platinum-containing (TP) regimen $(n=10)(p=0.097)$ (Fig. 2$)$. But the difference was not significant. To explore the role of systemic therapy in BM prevention, BMFS was also compared between regimens (patients with synchronous $\mathrm{BM}$ were excluded). Altogether, 38 patients received GP, 30 received TP, 16 received both GP and TP, 7 patients received TKIs (EGFR were unknown in 6 patients and mutated at exon 19 in 1 patient.) and 9 patients received other regimens (vinorelbine/platinum, cyclophosphamide/vinblastine/platinum, etoposide/platinum etc.). Survival analysis found that, patients who received both GP and TP before BM had the longest median BMFS than those who received other regimens $(p=0.013)$ (Fig. 3). Further analysis demonstrated that, the median BMFS was different significantly between those treated with both GP and TP and those treated with TP only $(p=0.009)$ or TKIs only ( $p=0.028)$, and marginally between those treated with both GP and TP and those treated with GP only ( $p=0.078)$. However, the difference in median BMFS between those treated with GP only and those treated with TP only was not significant $(p=0.10)$. 


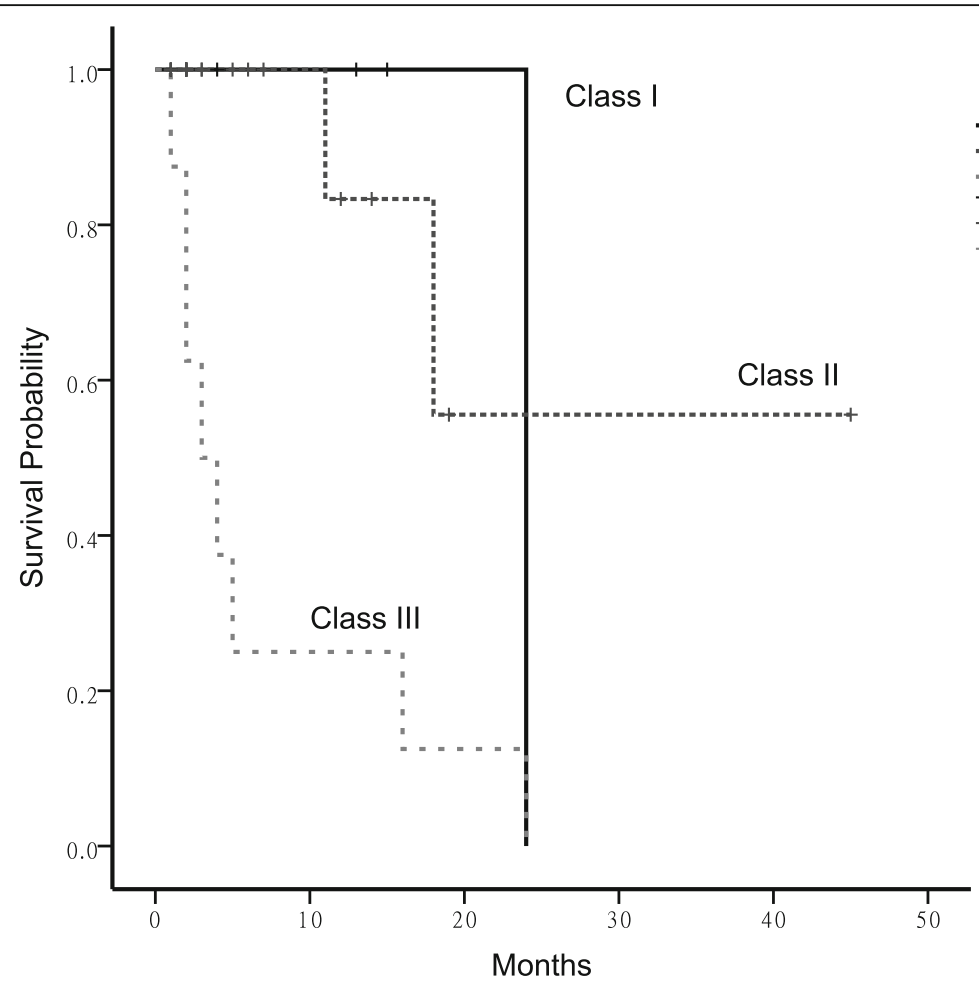

RPA

classification

$\neg$ Class I

- "Class II

- ". Class III

+Class I-censored

- Class II-censored

- Class III-censored

Fig. 1 Post brain metastasis survival by recursive partitioning analysis (RPA) classification $(p<0.001)$

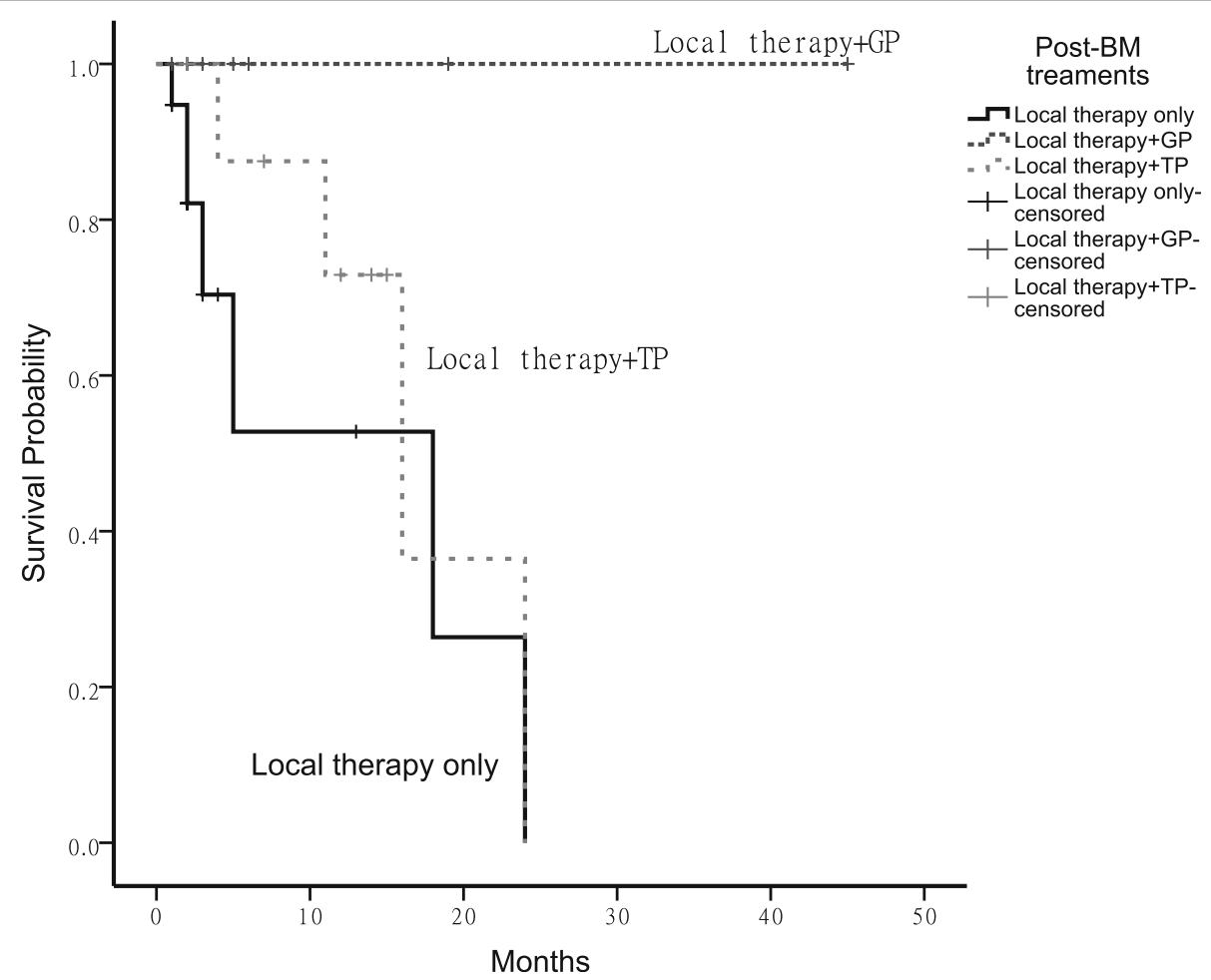

Fig. 2 Post brain metastasis survival by treatment after the occurrence of brain metastasis $(p=0.055)$ (local therapy only vs local therapy plus systemic therapy, $p=0.084$; local therapy plus GP vs local therapy plus TP, $p=0.097$ ) 
Table 3 Variants related to post-BM survival

\begin{tabular}{|c|c|c|c|c|c|}
\hline \multirow[t]{2}{*}{ Variants } & \multicolumn{2}{|l|}{ Single variant analysis } & \multicolumn{3}{|c|}{ Multivariate analysis } \\
\hline & Post-BM survival(months) & $P$ value & $\mathrm{HR}$ & $95 \% \mathrm{Cl}$ & $P$ value \\
\hline RPA & & $<0.001$ & 23.379 & $1.932-28.805$ & 0.013 \\
\hline । & 24 & & & & \\
\hline$\|$ & Not reached & & & & \\
\hline III & 3 & & & & \\
\hline GPA & & 0.087 & 2.895 & $0.435-19.285$ & 0.27 \\
\hline $0-1$ & 5 & & & & \\
\hline $1.5-2.5$ & 18 & & & & \\
\hline 3 & Not reached & & & & \\
\hline $3.5-4$ & Not reached & & & & \\
\hline Treatment after BM & & 0.084 & 0.058 & $0.008-0.410$ & 0.004 \\
\hline Local therapy only & 18 & & & & \\
\hline Local + systemic therapy & 24 & & & & \\
\hline
\end{tabular}

Abbreviations: BM Brain metastasis, RPA Recursive partitioning analysis, GPA Graded prognostic assessment

\section{Discussion}

Patients with SCC have lower incidence of BM. Some studies reported that, the incidence of $\mathrm{BM}$ in patients with SCC ranged from $8 \%$ to $28 \%$, which was lower than other histological subtypes such as adenocarcinoma, ranging from $20 \%$ to $45 \%[3,4,10,11]$. Additionally, previous studies concerning BM in NSCLC included different histological subtypes. As a result, the characteristics of BM in SCC patients have not been fully profiled. Similar to the reports of other studies, only $19.1 \%$ of patients in our study had documented BM over a decade. Therefore, it was a great challenge in releasing some convincing results with such relatively smaller samples. However, after careful analysis, there emerged some information which we believed to be worth noticing.

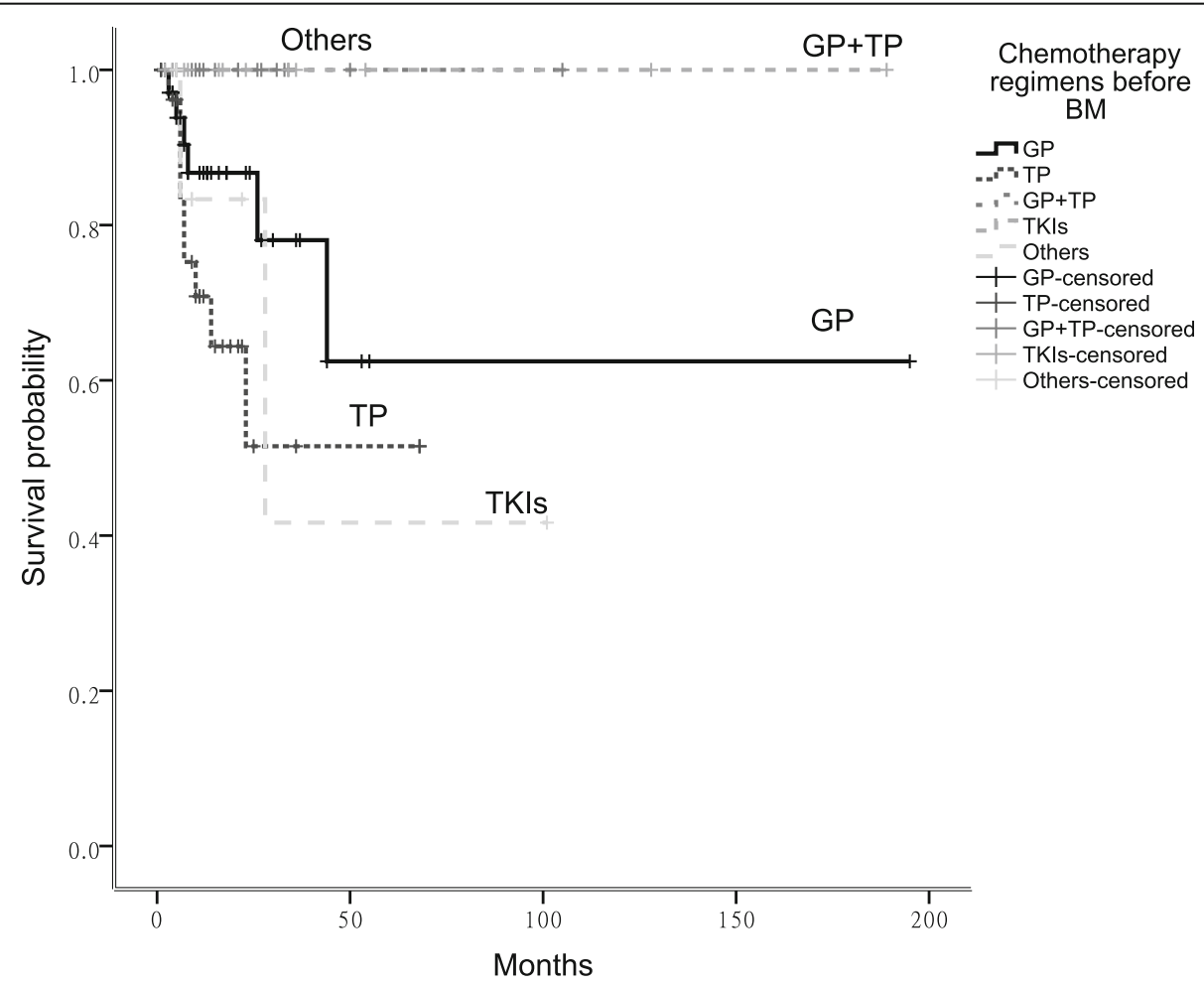

Fig. 3 Brain-metastasis-free survival by different regimens before the occurrence of brain metastasis $(p=0.013)$ 
We found that females, those with advanced disease, with adenosquamous histology and elevated serum level of CEA at diagnosis had a significantly higher incidence of BM. Besides, female and abnormal level of CEA were independent factors which indicated higher risk of BM. We are aware of two previous studies investigating the correlation between gender and BM [19, 20]. One showed no such correlation, but the other was in agreement with our findings. The underlying biology of these correlations is unknown. In our study, bivariate analysis found that female gender had a positive correlation with EGFR mutation $(p=0.024)$. In NSCLC, EGFR mutation is associated with constitutive activation of a downstream signaling pathway implicated in tumor cell growth, local invasion, angiogenesis and metastasis [21-23]. Some researchers have found that, a higher incidence of BM was seen in NSCLC patients with EGFR mutations than those with wild-type EGFR [16, 24, 25]. However, the evidence is stronger for adenocarcinoma than for SCC. In our study, among 33 patients with known EGFR status, only 5 SCC patients were shown to harbor EGFR mutations and none of them developed BM. So it is impossible to draw any conclusions as to whether EGFR mutation contributes to BM in SCC patients. Larger studies are needed to examine the role of EGFR mutation in BM. In addition, the role of other signal transduction pathways should be investigated.

CEA is the most commonly used biomarker for the screening of various malignancies including lung cancer. As it was shown in our study, patients with abnormal serum levels of CEA had not only higher incidence of $\mathrm{BM}$, but also higher risk of BM. In a perspective study enrolling 293 patients with staged IIIB-IV NSCLC also found, CEA $\geq 40 \mathrm{ng} / \mathrm{ml}$ was an independent risk factor of BM [26]. In another prospective study conduced by Lee et al., the association of tumor markers including CEA, Cyfra21-1, CA125, CA199 and SCC and BM were studied in 227 patients with advanced NSCLC [27]. As a result, CEA level was the only tumor marker that was found to be significantly different between patients with $\mathrm{BM}$ and without BM. Although some studies indicated that the prognostic value of CEA was more specific in adenocarcinoma patients than in SCC patients, it should be noticed that less than one third of patients enrolled in these studies had SCC histology [26, 28]. Since our findings were concluded in SCC patients, we believed that, at least in patients with SCC, those with abnormal serum levels of CEA at diagnosis should be considered as at risk population of BM. More concerns of BM should be paid to these patients during follow-up.

Some studies have suggested that NSCLC patients with local advanced disease have an increased risk of BM. This is confirmed in the current study, in which a higher stage at diagnosis is associated with a higher incidence of BM. Several clinical trials have therefore been carried out to explore the efficacy of PCI in patients with stage III disease $[1,2]$. However, while PCI decreased the incidence of BM, no survival advantage was shown. Data from our study showed that, the incidence of BM in patients with SCC and stage III disease was only $12.5 \%$. Even in studies of NSCLC which included patients with varying histological subtypes, the incidence of BM in stage III disease was not much higher, at $13-28 \%[3,4,11,20]$. Hence, stage was not qualified enough to define patients with higher risk of $\mathrm{BM}$, especially when used as single parameter. In addition to incidence, the interval from diagnosis to $\mathrm{BM}$ is another factor that should be considered. For NSCLC patients with local advanced disease, the median interval from diagnosis to BM ranges from 5.7 to 12 months [4, 19, 20, $29,30]$. Although some studies have shown that patients with squamous histology have longer intervals of BM [31], our study showed that the median time to BM for SCC patients with metachronous BM was only 7 months (range 5-8 months). This was much shorter than we expected. Furthermore, females and patients with elevated serum levels of CEA at diagnosis had a significantly shorter median BMFS than their counterparts. Thus, although patients with SCC have a lower incidence of BM, those with a higher risk of BM are likely to experience BM early in the course of their disease. Based on all these findings, it is reasonable that patients with higher risk of BM, such as female and patients with abnormal CEA at diagnosis indicated in our study should be the candidates of future studies concerning BM prevention. Furthermore, comprehensive studies are needed to further valid our findings. If possible, exploring risk factors other than clinical ones is also warranted.

RPA and GPA are prognostic systems for BM which includes many clinical factors such as age, Karnofsky performance status (KPS), number of BM and status of extracranial disease at the time of BM. In our study, RPA was found to be an independent prognostic factor for BM patients in multivariate analysis. More importantly, systemic therapy in addition to local therapy was also proved to be an independent prognostic factor which indicated better post-BM survival. Combined with the fact that BM was a kind of hematogeneous metastasis, it is warranted that systemic therapy should be considered after the occurrence of BM, especially for those accompanying extracranial metastasis. A standard systemic treatment regimen has not been established for SCC patients with BM. As a result of the JMDB study, in which subgroup analysis demonstrated superior efficacy of gemcitabine in patients with squamous histology, gemcitabine has become the optimal regimen for treating SCC patients [32]. Many studies have investigated the efficacy of platin-doublets involving a third generation cytotoxic drug as the first-line regimen 
for advanced NSCLC [33-35]. These trials have shown no survival benefit for any of these regimens, even when analyzed by histological subtype. In the current study, no significant survival difference was seen between those treated with GP and those treated with TP $(p=0.097)$, either. But we attribute the negative result more to smaller samples. Furthermore, we also compared median BMFS between different chemotherapy regimens to explore their ability to prevent BM. As a result, patients who received both GP and TP before BM had the longest median BMFS $(p=0.013)$. However, the difference between GP group and TP group was comparable $(p=0.10)$. This result suggests that BMFS may not agent-dependent. It may indicate that receiving both GP and TP prolongs BMFS or it may be that those who had a longer BMFS had the opportunity to receive both regimens. In terms of TKIs, since most patients in our study had unknown EGFR status, it is insufficient to evaluate their roles in the current setting. Thus, to date, the most effective agent for BM prevention or treatment in SCC patients remains uncertain.

\section{Conclusions}

To our knowledge, this is the first study to explore the risk factors for BM in SCC patients. It is true that our study was limited by retrospective study design and smaller samples of documented BM. But the factors we identified will be useful for the design of future studies to investigate preventative measures, such as PCI. Furthermore, we have shown that the addition of systemic therapy could significantly improve prognosis compared with local therapy alone. However, no agent was clearly superior for BM prevention or treatment in SCC patients.

\section{Abbreviations \\ BM: Brain metastasis; BMFS: Brain-metastasis-free survival; CEA: Carcinoembryonic antigen; Cyfra21-1: Cytokeratin 19 fragments; EGFR: Epidermal growth factor receptor; GPA: Graded prognostic assessment; KPS: Karnofsky performance status; NSCLC: Non-small cell lung cancer; NSE: Neuron-specific enolase; PBMS: Post-brain-metastasis survival; PCI: Prophylactic cranial irradiation; RPA: Recursive partitioning analysis; SCC: Squamous cell carcinoma; SCLC: Small cell lung cancer; SRS: Stereotactic radiosurgery; TKI: Tyrosine kinase inhibitor; WBRT: Whole brain radiation therapy}

\section{Acknowledgements}

Not applicable.

\section{Funding}

This work was supported by the Capital Foundation of Medical Development (Grant number: 2014-4-5082) and the Project supported by Beijing Postdoctoral Research Foundation.

\section{Availability of data and materials}

The datasets used and/or analysed during the current study available from the corresponding author on reasonable request.

\section{Authors' contributions}

LB conceived of the study, participated in its design, performed the statistical analysis and drafted the manuscript. LY and LS participated in the data collection and statistical analysis. QX conceived of the study, and participated in its design and coordination. All authors read and approved the final manuscript.
Ethics approval and consent to participate

The study was reviewed and approved by the institutional review boards and ethics committees of Beijing Tiantan Hospital affiliated to Capital Medical University. (KY2014-033-01).

Consent for publication

Not applicable

\section{Competing interests}

The authors declare that they have no competing interests.

Received: 18 November 2016 Accepted: 17 August 2017

Published online: 07 November 2017

\section{References}

1. Pottgen C, Eberhardt W, Grannass A, Korfee S, Stuben G, et al. Prophylactic cranial irradiation in operable stage IIIA non small-cell lung cancer treated with neoadjuvant chemoradiotherapy: results from a German multicenter randomized trial. J Clin Oncol. 2007;25:4987-92.

2. Gore EM, Bae K, Wong SJ, Sun A, Bonner JA. Phase III comparison of prophylactic cranial irradiation versus observation in patients with locally advanced non-small-cell lung cancer: primary analysis of radiation therapy oncology group study RTOG 0214. J Clin Oncol. 2011;29:272-8.

3. Ceresoli GL, Reni M, Chiesa G, Carretta A, Schipani S, et al. Brain metastases in locally advanced nonsmall cell lung carcinoma after multimodality treatment: risk factors analysis. Cancer. 2002;95:605-12.

4. Robnett TJ, Machtay M, Stevenson JP, Algazy KM, Hahn SM. Factors affecting the risk of brain metastases after definitive chemoradiation for locally advanced non-small-cell lung carcinoma. J Clin Oncol. 2001;19:1344-9.

5. Sperduto PW, Berkey B, Gaspar LE, Mehta M, Curran W. A new prognostic index and comparison to three other indices for patients with brain metastases: an analysis of 1,960 patients in the RTOG database. Int J Radiat Oncol Biol Phys. 2008;70:510-4.

6. Chang DB, Yang PC, Luh KT, Kuo SH, Hong RL, et al. Late survival of nonsmall cell lung cancer patients with brain metastases. Influence of treatment Chest. 1992;101:1293-7.

7. Gaspar L, Scott C, Rotman M, Asbell S, Phillips T, et al. Recursive partitioning analysis (RPA) of prognostic factors in three radiation therapy oncology group (RTOG) brain metastases trials. Int J Radiat Oncol Biol Phys. 1997;37:745-51.

8. Aupérin A, Arriagada R, Pignon JP, Le Péchoux C, Gregor A, et al. Prophylactic cranial irradiation for patients with small-cell lung cancer in complete remission. Prophylactic cranial irradiation overview collaborative group. N Engl J Med. 1999;341:476-84.

9. Meert AP, Paesmans M, Berghmans T, Martin B, Mascaux C, et al. Prophylactic cranial irradiation in small cell lung cancer: a systematic review of the literature with meta-analysis. BMC Cancer. 2001;1:5.

10. Cox JD, Scott CB, Byhardt RW, Emami B, Russell AH, et al. Addition of chemotherapy to radiation therapy alters failure patterns by cell type within non-small cell carcinoma of lung (NSCCL): analysis of radiation therapy oncology group (RTOG) trials. Int J Radiat Oncol Biol Phys. 1999;43:505-9.

11. Wang SY, Ye X, Ou W, Lin YB, Zhang BB, et al. Risk of cerebral metastases for postoperative locally advanced non-small-cell lung cancer. Lung Cancer. 2009;64:238-43.

12. Gavrilovic IT, Posner JB. Brain metastases: epidemiology and pathophysiology. J Neuro-Oncol. 2005;75:5-14.

13. Andre F, Grunenwald D, Pujol JL, Girard P, Dujon A, et al. Patterns of relapse of N2 nonsmall-cell lung carcinoma patients treated with preoperative chemotherapy: should prophylactic cranial irradiation be reconsidered? Cancer. 2001;91:2394-400.

14. Zeng YD, Zhang L, Liao H, Liang $Y, X u F$, et al. Gefitinib alone or with concomitant whole brain radiotherapy for patients with brain metastasis from non-small-cell lung cancer: a retrospective study. Asian Pac J Cancer Prev. 2012;13:909-14.

15. Park SJ, Kim HT, Lee DH, Kim KP. Efficacy of epidermal growth factor receptor tyrosine kinase inhibitors for brain metastasis in non-small cell lung cancer patients harboring either exon 19 or 21 mutation. Lung Cancer. 2012:77:556-60.

16. Welsh JW, Komaki R, Amini A, Munsell MF, Unger W, et al. Phase II trial of erlotinib plus concurrent whole-brain radiation therapy for patients with brain metastases from non-small-cell lung cancer. J Clin Oncol. 2013;31:895-902. 
17. Bailon O, Chouahnia K, Augier A, Bouillet T, Billot $\mathrm{S}$, et al. Upfront association of carboplatin plus pemetrexed in patients with brain metastases of lung adenocarcinoma. Neuro-Oncology. 2012;14:491-5.

18. Barlesi F, Gervais $\mathrm{R}$, Lena $\mathrm{H}$, Hureaux J, Berard $\mathrm{H}$, et al. Pemetrexed and cisplatin as first-line chemotherapy for advanced non-small-cell lung cancer (NSCLC) with asymptomatic inoperable brain metastases: a multicenter phase II trial (GFPC 07-01). Ann Oncol. 2011;22:2466-70.

19. Bajard A, Westeel V, Dubiez A, Jacoulet P, Pernet $D$, et al. Multivariate analysis of factors predictive of brain metastases in localised non-small cell lung carcinoma. Lung Cancer. 2004;45:317-23.

20. Ji Z, Bi N, Wang J, Hui Z, Xiao Z, et al. Risk factors for brain metastases in locally advanced non-small cell lung cancer with definitive chest radiation. Int J Radiat Oncol Biol Phys. 2014;89:330-7.

21. Politi K, Zakowski MF, Fan PD, Schonfeld EA, Pao W, et al. Lung adenocarcinomas induced in mice by mutant EGF receptors found in human lung cancers respond to a tyrosine kinase inhibitor or to down-regulation of the receptors. Genes Dev. 2006;20:1496-510.

22. Okabe T, Okamoto I, Tamura K, Terashima M, Yoshida T, et al. Differential constitutive activation of the epidermal growth factor receptor in non-small cell lung cancer cells bearing EGFR gene mutation and amplification. Cancer Res. 2007;67:2046-53.

23. Lynch TJ, Bell DW, Sordella R, Gurubhagavatula S, Okimoto RA, et al. Activating mutations in the epidermal growth factor receptor underlying responsiveness of non-small-cell lung cancer to gefitinib. N Engl J Med. 2004;350:2129-39.

24. Shin DY, Na II, Kim CH, Park S, Baek H, et al. EGFR mutation and brain metastasis in pulmonary adenocarcinomas. J Thorac Oncol. 2014;9:195-9.

25. Li B, Sun SZ, Yang M, Shi JL, Xu W, et al. The correlation between EGFR mutation status and the risk of brain metastasis in patients with lung adenocarcinoma. J Neuro-Oncol. 2015;124:79-85.

26. Arrieta O, Saavedra-Perez D, Kuri R, Aviles-Salas A, Martinez L, et al. Brain metastasis development and poor survival associated with carcinoembryonic antigen (CEA) level in advanced non-small cell lung cancer: a prospective analysis. BMC Cancer. 2009;9:119.

27. Lee DS, Kim YS, Jung SL, Lee KY, Kang JH, et al. The relevance of serum carcinoembryonic antigen as an indicator of brain metastasis detection in advanced non-small cell lung cancer. Tumour Biol. 2012;33:1065-73.

28. Okada M, Nishio W, Sakamoto T, Uchino K, Yuki T, et al. Effect of histologic type and smoking status on interpretation of serum carcinoembryonic antigen value in non-small cell lung carcinoma. Ann Thorac Surg. 2004;78: 1004-9.

29. Chen AM, Jahan TM, Jablons DM, Garcia J, Larson DA. Risk of cerebral metastases and neurological death after pathological complete response to neoadjuvant therapy for locally advanced nonsmall-cell lung cancer: clinical implications for the subsequent management of the brain. Cancer. 2007; 109:1668-75.

30. Carolan H, Sun AY, Bezjak A, Yi QL, Payne D, et al. Does the incidence and outcome of brain metastases in locally advanced non-small cell lung cancer justify prophylactic cranial irradiation or early detection? Lung Cancer. 2005; 49:109-15.

31. Gaspar LE, Chansky K, Albain KS, Vallieres E, Rusch V, et al. Time from treatment to subsequent diagnosis of brain metastases in stage III nonsmall-cell lung cancer: a retrospective review by the southwest oncology group. J Clin Oncol. 2005;23:2955-61.

32. Scagliotti GV, Parikh P, von Pawel J, Biesma B, Vansteenkiste J, et al. Phase II study comparing cisplatin plus gemcitabine with cisplatin plus pemetrexed in chemotherapy-naive patients with advanced-stage non-small-cell lung cancer. J Clin Oncol. 2008;26:3543-51.

33. Hoang T, Dahlberg SE, Schiller JH, Johnson DH. Does histology predict survival of advanced non-small cell lung cancer patients treated with platinbased chemotherapy? An analysis of the eastern cooperative oncology group study E1594. Lung Cancer. 2013;81:47-52.

34. Scagliotti GV, De Marinis F, Rinaldi M, Crino L, Gridelli C, et al. Phase III randomized trial comparing three platinum-based doublets in advanced non-small-cell lung cancer. J Clin Oncol. 2002;20:4285-91.

35. Scagliotti GV, De Marinis F, Rinaldi M, Crino L, Gridelli C, et al. The role of histology with common first-line regimens for advanced non-small cell lung cancer: a brief report of the retrospective analysis of a three-arm randomized trial. J Thorac Oncol. 2009;4:1568-71.

\section{Submit your next manuscript to BioMed Central and we will help you at every step:}

- We accept pre-submission inquiries

- Our selector tool helps you to find the most relevant journal

- We provide round the clock customer support

- Convenient online submission

- Thorough peer review

- Inclusion in PubMed and all major indexing services

- Maximum visibility for your research

Submit your manuscript at www.biomedcentral.com/submit
C Biomed Central 